\title{
KANTBP 4M Program for Solving the Scattering Problem for a System of Ordinary Second-Order Differential Equations
}

\author{
Galmandakh Chuluunbaatar ${ }^{1,2}$, Alexander A. Gusev ${ }^{1, \star}$, Ochbadrakh Chuluunbaatar ${ }^{1,3, \star \star}$, \\ Sergue I. Vinitsky ${ }^{1,2, \star \star \star}$, and Luong Le Hai ${ }^{4}$ \\ ${ }^{1}$ Joint Institute for Nuclear Research, Dubna, Russia \\ ${ }^{2} R U D N$ University, Moscow, Russia \\ ${ }^{3}$ Institute of Mathematics, National University of Mongolia, Ulaanbaatar, Mongolia \\ ${ }^{4}$ Ho Chi Minh city University of Education, Ho Chi Minh city, Vietnam
}

\begin{abstract}
We report an upgrade of the program KANTBP 4M implemented in the computer algebra system MAPLE for solving, with a given accuracy, the multichannel scattering problem, which is reduced to a boundary-value problem for a system of ordinary differential equations of the second order with continuous or piecewise continuous real or complex-valued coefficients. The solution over a finite interval is subject to mixed homogeneous boundary conditions: Dirichlet and/or Neumann, and/or of the third kind. The discretization of the boundary problem is implemented by means of the finite element method with the Lagrange or Hermite interpolation polynomials. The efficiency of the proposed algorithm is demonstrated by solving a multichannel scattering problem with coupling of channels in both the reaction region and the asymptotic one.
\end{abstract}

\section{Introduction}

A wide class of quantum mechanical problems such as tunneling, ionization, recombination problem, are formulated as boundary-value problems (BVPs) for elliptic differential equations. One of the ways to solve these problems is to reduce them to a multichannel scattering problem for a set of secondorder ordinary differential equations (SOODEs) in an infinite region (on axis or semiaxis), which can be performed by different methods. Further reduction of the problem to a finite interval implies the formulation of asymptotic boundary conditions depending upon the kind of the physical problem considered.

For the general multichannel scattering problem, the nonsymmetry in the coefficient functions leads to the different numbers of open channels in the left-hand and right-hand asymptotic regions, i.e., a scattering matrix composed of square matrices of reflection amplitudes and rectangular matrices of transmission amplitudes [1]. To solve this problem, as well as the problems of bound or metastable states [2], we elaborated a new version of the program KANTBP 4M [3] implementing a finite element method (FEM) with Lagrange or Hermite interpolation polynomials [4] in the computer algebra system (CAS) MAPLE.

\footnotetext{
^e-mail: gooseff@jinr.ru

$\star \star$ e-mail: chuka@jinr.ru

$\star \star \star$ e-mail: vinitsky@theor.jinr.ru
} 
In the present paper we modify the algorithms to solve the scattering problem with coupled ODEs in both the reaction regions and the asymptotic ones, implemented in an upgraded version of the program KANTBP 4M. The efficiency of the proposed algorithm is demonstrated by solving a multichannel scattering problem with coupling of channels in the asymptotic region similar to the one present in sub-barrier fusion reactions [6].

\section{The multichannel scattering problem}

The program KANTBP $4 \mathrm{M}$ is intended for solving the BVPs for the system of SOODEs with respect to the unknown functions $\boldsymbol{\Phi}(z)=\left(\Phi_{1}(z), \ldots, \Phi_{N}(z)\right)^{T}$ of the independent variable $z \in \Omega=\left(z^{\min }, z^{\max }\right)$ :

$$
\left(-\frac{1}{f_{B}(z)} \mathbf{I} \frac{d}{d z} f_{A}(z) \frac{d}{d z}+\mathbf{V}(z)+\frac{f_{A}(z)}{f_{B}(z)} \mathbf{Q}(z) \frac{d}{d z}+\frac{1}{f_{B}(z)} \frac{d f_{A}(z) \mathbf{Q}(z)}{d z}-E \mathbf{I}\right) \mathbf{\Phi}(z)=0 .
$$

Here $f_{B}(z)>0$ and $f_{A}(z)>0$ are continuous or piecewise continuous positive functions, $\mathbf{I}$ is the unit matrix, $\mathbf{V}(z)$ is a symmetric matrix, $V_{i j}(z)=V_{j i}(z)$, and $\mathbf{Q}(z)$ is an antisymmetric matrix, $Q_{i j}(z)=$ $-Q_{j i}(z)$, of the effective potentials having the dimension $N \times N$. The elements of these matrices are continuous or piecewise continuous real or complex-valued coefficients from the Sobolev space $\mathcal{H}_{2}^{s \geq 1}(\Omega)$, providing the existence of nontrivial solutions subject to mixed homogeneous boundary conditions: Dirichlet and/or Neumann, and/or of the third kind at the boundary points of the interval $z \in\left[z^{\min }, z^{\max }\right]$ at given values of the elements of the real or complex-valued $N \times N$ matrix $\mathbf{G}\left(z^{t}\right)=$ $\mathcal{R}\left(z^{t}\right)-\mathbf{Q}\left(z^{t}\right)$ at $z^{t}=z^{\min }$ or $z^{t}=z^{\max }$ :

$$
\text { (I): } \boldsymbol{\Phi}\left(z^{t}\right)=0 \text {, (II): } \lim _{z \rightarrow z^{t}} f_{A}(z)\left(\mathbf{I} \frac{d}{d z}-\mathbf{Q}(z)\right) \boldsymbol{\Phi}(z)=0 \text {, (III): } \lim _{z \rightarrow z^{t}} f_{A}(z)\left(\mathbf{I} \frac{d}{d z}-\mathbf{Q}(z)-\mathbf{G}(z)\right) \boldsymbol{\Phi}(z)=0 \text {. }
$$

For the multichannel scattering problem at fixed energy $E \equiv \mathfrak{R} E$ the desired matrix of solutions $\boldsymbol{\Phi}(z)$ is calculated with the asymptotic form "incident wave + outgoing waves"

$$
\begin{gathered}
\boldsymbol{\Phi}_{\rightarrow}(z \rightarrow \pm \infty)= \begin{cases}\mathbf{X}_{\max }^{(\rightarrow)}(z) \mathbf{T}_{\rightarrow}+\mathbf{X}_{\max }^{(c)}(z) \mathbf{T}_{\rightarrow}^{c}, & z>0, \\
\mathbf{X}_{\min }^{(\rightarrow)}(z)+\mathbf{X}_{\min }^{(\leftarrow)}(z) \mathbf{R}_{\rightarrow}+\mathbf{X}_{\min }^{(c)}(z) \mathbf{R}_{\rightarrow}^{c}, & z<0,\end{cases} \\
\boldsymbol{\Phi}_{\leftarrow}(z \rightarrow \pm \infty)= \begin{cases}\mathbf{X}_{\max }^{(\leftarrow)}(z)+\mathbf{X}_{\max }^{(\rightarrow)}(z) \mathbf{R}_{\leftarrow}+\mathbf{X}_{\max }^{(c)}(z) \mathbf{R}_{\leftarrow}^{c}, & z>0, \\
\mathbf{X}_{\min }^{(\leftarrow)}(z) \mathbf{T}_{\leftarrow}+\mathbf{X}_{\min }^{(c)}(z) \mathbf{T}_{\leftarrow}^{c}, & z<0 .\end{cases}
\end{gathered}
$$

The desired quantities are the square $N_{o}^{L} \times N_{o}^{L}$ and $N_{o}^{R} \times N_{o}^{R}$ matrices $\mathbf{R}_{\rightarrow}$ and $\mathbf{R}_{\leftarrow}$ of reflection amplitudes and the rectangular $N_{o}^{R} \times N_{o}^{L}$ and $N_{o}^{L} \times N_{o}^{R}$ matrices $\mathbf{T}_{\rightarrow}$ and $\mathbf{T}_{\leftarrow}$ of transmission amplitudes, where $N_{o}^{L}$ and $N_{o}^{R}$ denote numbers of open channels in the asymptotic regions. The coefficients of Eq. (1) in the asymptotic regions $z \leq z^{\min }$ or $z \geq z^{\max }$ satisfy the conditions:

$$
f_{B}(z) / f_{A}^{t}=1+o(1), \quad V_{i j}(z)=V_{i j}^{t}+o(1), \quad Q_{i j}(z)=o(1) .
$$

Here the label $t$ marks the asymptotic region: $t=L$ at $z \leq z^{\min }$ and $t=R$ at $z \geq z^{\max }$. In this case the expressions (3)-(4) are written in the explicit form

$$
\begin{aligned}
& \boldsymbol{\Phi}_{i o}^{(\rightarrow)}(z \rightarrow-\infty)=\sum_{i=1}^{N} \boldsymbol{\Psi}_{i}^{(L)} \begin{cases}\frac{\exp \left(\iota \sqrt{E-\lambda_{i}^{(L)}} z\right)}{\sqrt[4]{E-\lambda_{i}^{(L)}}} \delta_{i i i_{o}^{L}}+R_{i i i_{o}^{L}} \frac{\exp \left(-\imath \sqrt{E-\lambda_{i}^{(L)}} z\right)}{\sqrt[4]{E-\lambda_{i}^{(L)}}}, & E>\lambda_{i}^{(L)}, \\
R_{i i_{o}^{L}}^{c} \exp \left(\sqrt{\lambda_{i}^{(L)}-E} z\right), & E \leq \lambda_{i}^{(L)},\end{cases} \\
& \boldsymbol{\Phi}_{i_{o}^{L}}^{(\rightarrow)}(z \rightarrow+\infty)=\sum_{i=1}^{N} \boldsymbol{\Psi}_{i}^{(R)} \begin{cases}T_{i i_{o}^{L}} \frac{\exp \left(\iota \sqrt{E-\lambda_{i}^{(R)}} z\right)}{\sqrt[4]{E-\lambda_{i}^{(R)}}}, & E>\lambda_{i}^{(R)}, \\
T_{i i_{o}^{L}}^{c} \exp \left(-\sqrt{\lambda_{i}^{(R)}-E} z\right), & E \leq \lambda_{i}^{(R)},\end{cases}
\end{aligned}
$$




$$
\begin{gathered}
\boldsymbol{\Phi}_{i_{o}^{R}}^{(\leftarrow)}(z \rightarrow-\infty)=\sum_{i=1}^{N} \boldsymbol{\Psi}_{i}^{(L)} \begin{cases}T_{i i_{o}^{R}} \frac{-\exp \left(\iota \sqrt{E-\lambda_{i}^{(L)}} z\right)}{\sqrt[4]{E-\lambda_{i}^{(L)}}}, & E>\lambda_{i}^{(L)}, \\
T_{i i_{o}^{R}}^{c} \exp \left(\sqrt{\lambda_{i}^{(L)}-E} z\right), & E \leq \lambda_{i}^{(L)},\end{cases} \\
\boldsymbol{\Phi}_{i_{o}^{R}}^{(\leftarrow)}(z \rightarrow+\infty)=\sum_{i=1}^{N} \boldsymbol{\Psi}_{i}^{(R)} \begin{cases}\frac{\exp \left(-\imath \sqrt{E-\lambda_{i}^{(R)}} z\right)}{\sqrt[4]{E-\lambda_{i}^{(R)}}} \delta_{i i_{o}^{R}}+R_{i i i_{o}^{R}} \frac{\exp \left(\iota \sqrt{E-\lambda_{i}^{(R)}} z\right)}{\sqrt[4]{E-\lambda_{i}^{(R)}},}, & E>\lambda_{i}^{(R)}, \\
R_{i i_{o}^{R}}^{c} \exp \left(-\sqrt{\lambda_{i}^{(R)}-E} z\right), & E \leq \lambda_{i}^{(R)} .\end{cases}
\end{gathered}
$$

Here $\lambda_{i}^{(t)}$ and $\Psi_{i}^{(t)}=\left\{\Psi_{1 i}^{(t)}, \ldots, \Psi_{N i}^{(t)}\right\}^{T}$ are the solutions of the algebraic eigenvalue problem

$$
\mathbf{V}_{m}^{t} \boldsymbol{\Psi}_{i}^{(t)}=\lambda_{i}^{(t)} \boldsymbol{\Psi}_{i}^{(t)}, \quad\left(\boldsymbol{\Psi}_{i}^{(t)}\right)^{T} \boldsymbol{\Psi}_{j}^{(t)}=\delta_{i j}
$$

The details of the FEM solution of the above problems are given in Refs. [2, 3].

\section{A nontrivial case study}

We consider the multichannel scattering problem for the Schrödinger equation in the 2D domain $\Omega_{y z}=\{y \in(0, \pi), z \in(-\infty,+\infty)\}$, with the potential $V(y, z)=\{0, z<2 ;-2 y,|z| \leq 2 ; 2 y, z>2\}$. We seek the solution in the form of the expansion $\Psi(y, z)=\sum_{i=1}^{N} B_{i}(y) \Phi_{i}(z)$ in the set of basis functions $B_{i}(y)=$ $\sqrt{2 / \pi} \sin (i y)$, leading to the system (1) with $f_{B}(z)=f_{A}(z)=1, Q_{i j}(z)=0$ and the effective potentials

$$
V_{i j}(z)=i^{2} \delta_{i j}+\{0, z<-2 ;-2,|z| \leq 2 ; 2, z>2\} \times\left\{\pi / 2, i=j ; 0 \text {, even } i-j ; \frac{-8 i j}{\pi\left(i^{2}-j^{2}\right)^{2}} \text {, odd } i-j\right\} \text {. }
$$

The considered system at $N=6$ has sets of threshold energies that are different for the left- and righthand asymptotic regions of the $z$-axis: $\lambda_{i}^{(L)}=\{1,4,9,16,25,36\}$ and $\lambda_{i}^{(R)}=\{3.742260,7.242058$, $12.216485,19.188688,28.173689,39.286376\}$. Here $\lambda_{i}^{(R)}$ are eigenvalues of the algebraic eigenvalue problem (10) corresponding to the eigenvectors:

$$
\boldsymbol{\Psi}_{i}^{(R)}=\left(\begin{array}{rrrrrr}
-0.942058 & 0.333910 & -0.031282 & 0.007167 & 0.000688 & 0.000744 \\
-0.331595 & -0.912914 & 0.237277 & -0.017164 & -0.006040 & -0.000623 \\
-0.049715 & -0.233531 & -0.955002 & 0.175590 & 0.010339 & 0.004970 \\
-0.009739 & -0.022620 & -0.174676 & -0.974511 & -0.138406 & -0.007390 \\
-0.002119 & -0.006461 & -0.012475 & -0.138177 & 0.983791 & 0.113406 \\
-0.000916 & -0.000915 & -0.005074 & -0.007629 & 0.113382 & -0.993508
\end{array}\right) .
$$

The solutions of the BVPs for the system of ODEs (1) with piecewise constant potential (11) can be obtained by the method of matching the fundamental solutions (MMFS) (see Ref. [5]), that is used as a test for the algorithm and the programm KANTBP 4M, implementing the FEM in the CAS Maple [3].

At given $E=6$ for the wave incident from the left there are two open channels $N_{o}^{L}=2$ and for the wave incident from the right there is one open channel $N_{o}^{R}=1$. The desired $\mathbf{S}$-matrix takes the form

$$
\mathbf{S}=\left(\begin{array}{ll}
\mathbf{R}_{\rightarrow} & \mathbf{T}_{\leftarrow} \\
\mathbf{T}_{\rightarrow} & \mathbf{R}_{\leftarrow}
\end{array}\right)=\left(\begin{array}{rr|r}
0.358853+0.766130 i & -0.281552-0.006531 i & -0.242783-0.382115 i \\
-0.281552-0.006531 i & 0.084039-0.485524 i & -0.803553+0.179421 i \\
\hline-0.242783-0.382115 i & -0.803553+0.179421 i & -0.170539-0.296767 i
\end{array}\right),
$$

where the square $\mathbf{R}_{\rightarrow}, \mathbf{R}_{\leftarrow}$ and rectangular $\mathbf{T}_{\rightarrow}, \mathbf{T}_{\leftarrow}$ submatrices are separated by lines. The corresponding wave functions of the multichannel scattering problem are presented in Fig. 1.

The FEM calculations were performed in intervals $z \in[-2,2]$ and $z \in[-10,10]$ with seventh order Hermite elements $\left(p^{\prime}=7, p=3, \kappa^{\max }=2\right)$ on the finite element meshes $\Omega_{z}=\{-2(4) 2\}$ and $\Omega_{z}=\{-10(6)-2(4) 2(6) 10\}$, respectively, where in the parentheses the number of elements is indicated. The results obtained by FEM in the above intervals and MMFS coincide within $10^{-10}$. 


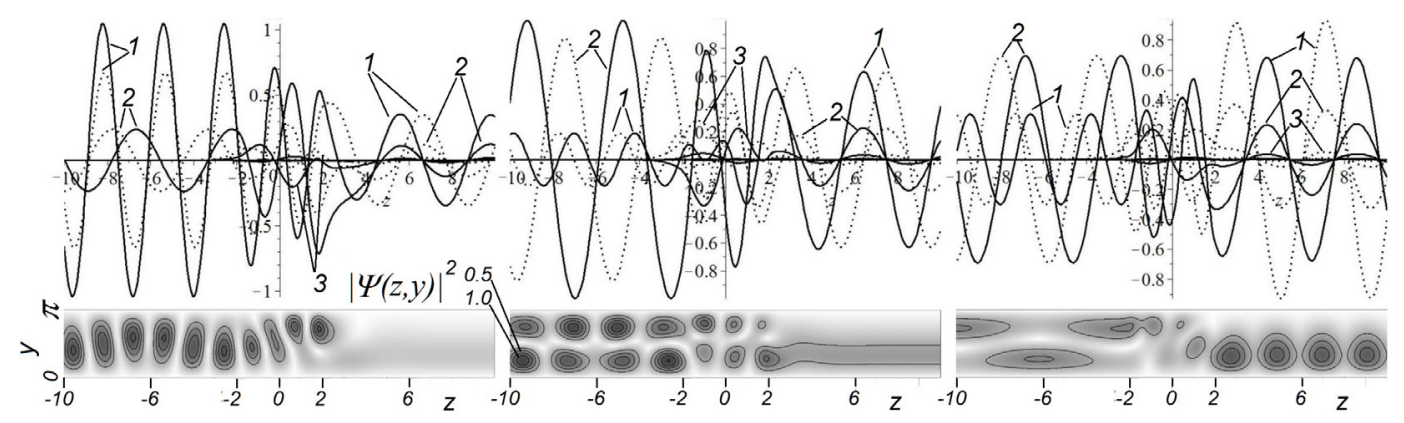

Figure 1. Real (solid lines) and imaginary (dotted lines) components $\Phi_{i}$ (labeled by $i=1,2,3$ ) of the solution $\boldsymbol{\Phi}$ of the multichannel scattering problem for the waves, incident from the left from the first, LR(1) and the second LR(2) open channels, and the wave incident from the right RL(1), and profiles of square of absolute values $|\Psi(y, z)|^{2}$ of the corresponding $2 \mathrm{D}$ wave functions $\Psi(y, z)$.

\section{Conclusions}

The KANTBP 4M code and the test examples of solving eigenvalue and scattering problems of quantum mechanics are available in the program library JINRLIB [3]. The upgrade of KANTBP 4M program implemented in the CAS MAPLE for solving with a given accuracy the boundary value problems for systems of coupled SOODEs, in particular, the class of scattering problem for the system of coupled SOODEs in both the reaction regions and the asymptotic ones with different number of open channels in the left-hand and right-hand coupled asymptotic regions is approbated. The Fortran version of KANTBP $4 \mathrm{M}$ program that allows one to perform large scale calculations of the sub-barrier fusion reactions of heavy ions will be published elsewhere.

\section{Acknowledgements}

The work was partially supported by the RFBR (grant No 18-51-18005), the Bogoliubov-Infeld and Hulubei-Meshcheryakov programs, the Plenipotentiary Representative of the Government of the Republic of Kazakhstan in the framework of collaboration program JINR-RK, and the Ho Chi Minh city University of Education (grant CS.2018.19.50). The publication has been prepared under partial support from the "RUDN University Program 5-100".

\section{References}

[1] A.A. Gusev, et al., Computer Physics Communications 185, 3341-3343 (2014)

[2] A.A. Gusev, et al., Lecture Notes in Computer Science 9301, 182-197 (2015)

[3] A.A. Gusev, et al., JINRLIB [https://www1.jinr.ru/programs/jinrlib/kantbp4m/indexe.html]

[4] A.A. Gusev, et al., Lecture Notes in Computer Science 8660, 138-154 (2014)

[5] A.A. Gusev, et al., Discrete and Continuous Models and Applied Computational Science, 3, 38-52 (2016)

[6] P.W. Wen, et al., in book of abstracts of LXIX International Conference "NUCLEUS-2019", Dubna, Russia, 1-5 July, 2019, p. 294. 